\title{
Spontaneous current generation in gated nanostructures
}

\author{
D. W. Horsell, ${ }^{1}$ A. K. Savchenko, ${ }^{1}$ Y. M. Galperin, ${ }^{2,3,4}$ V. I. Kozub,${ }^{3,4}$ V. M. Vinokur, ${ }^{4}$ and D. A. Ritchie ${ }^{5}$ \\ ${ }^{1}$ School of Physics, University of Exeter, Exeter, EX4 4QL, UK \\ ${ }^{2}$ Department of Physics, University of Oslo, PO Box 1048 Blindern, 0316 Oslo, Norway \\ ${ }^{3}$ A. F. Ioffe Physico-Technical Institute of Russian Academy of Sciences, 194021 St. Petersburg, Russia \\ ${ }^{4}$ Argonne National Laboratory, 9700 S. Cass av., Argonne, IL 60439, USA \\ ${ }^{5}$ Cavendish Laboratory, University of Cambridge, Cambridge, CB3 OHE, UK
}

\begin{abstract}
We have observed an unusual dc current spontaneously generated in the conducting channel of a short-gated GaAs transistor. The magnitude and direction of this current critically depend upon the voltage applied to the gate. We propose that it is initiated by the injection of hot electrons from the gate that relax via phonon emission. The phonons then excite secondary electrons from asymmetrically distributed impurities in the channel, which leads to the observed current.
\end{abstract}

PACS numbers: 72.20.-i, 72.40.+w, 72.90.+y, 73.23.-b

Gated semiconductor nanostructures have become the staple diet of modern condensed matter research and applications. Their small size has resulted in a wealth of new phenomena observed in electron transport, including universal conductance fluctuations [1, 2] and the photogalvanic effect [3, 4]. In such a structure, at low temperatures we observe a dc current through the conducting channel in the absence of any applied bias. This current is dependent upon the gate voltage $V_{g}$, which dictates its magnitude and direction through the channel.

It was found that the observed current could not be produced by conventional sources of residual bias and stray interference coupling to the system [5, [6]. We propose a model that eliminates this apparent "Maxwell's demon" required to support the voltage across the sample. A small gate leakage current is magnified in the source-drain circuit due to phonon-assisted excitation of localized electrons. While the leakage current itself has a smooth dependence on $V_{g}$, the "spontaneous" current changes its direction due to the $V_{g}$-dependent asymmetry of the channel. It transpires that the effect is greatest for channels of length $\sim 0.1 \mu \mathrm{m}$, which is the key size in contemporary nanostructures.

The experiments were carried out on a GaAs based transistor. The wafer consists of a $1 \cdot 10^{17} \mathrm{~cm}^{-3}$ silicon doped layer $1450 \AA$ thick on an undoped GaAs substrate. A metallic $(\mathrm{Au})$ gate, of length $0.15 \mu \mathrm{m}$ in the current direction and width $9 \mu \mathrm{m}$, was formed between the source and drain, see Fig. 1(inset). For large negative gate voltages the two-terminal conductance of the device is dominated by the region under the gate; this region defines the "channel", and regions outside the gate are the "contacts". Measurements were carried out in a dilution refrigerator at a base temperature of $30 \mathrm{mK}$, housed in a screen room to suppress external interference. Both the ac and dc currents were measured via a battery-powered EG\&G 181 low-noise pre-amplifier within the room.

Figure 1 shows the conductance $(\mathrm{d} i / \mathrm{d} V)$ through the channel as a function of the applied gate voltage. Strong, reproducible structure can be seen to occur near the

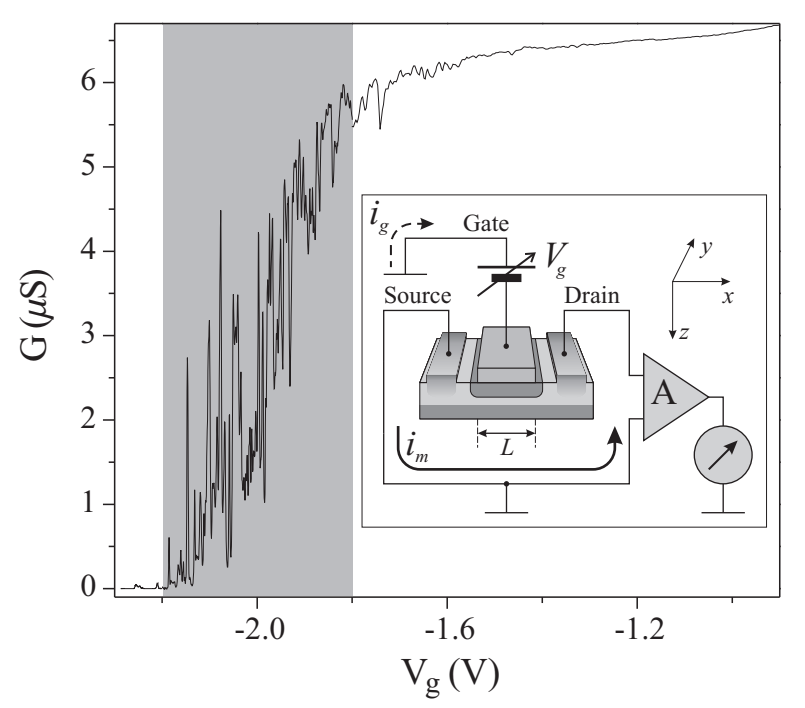

FIG. 1: The two-terminal (differential) conductance as a function of the gate voltage. The shaded box delimits the range of $V_{g}$ where the spontaneous current is resolved. Inset: Circuit (simplified) used to measure the dc current $i_{m}$ via the pre-amplifier (A). The transistor is depicted schematically: conductive regions are shown in light grey, and depleted regions in dark grey.

pinch-off, associated with mesoscopic hopping and tunnelling processes [7]. In the absence of a voltage source in the source-drain circuit, shown in Fig. 1(inset), one would expect the measured dc current $i_{m}$ to be zero at any gate voltage. Contrarily, Fig. 2(a) shows that a large current occurs that changes direction and magnitude as a function of $V_{g}$. This current is only resolved in a small range of gate voltages, highlighted in Fig. 1, where fluctuations of the conductance are large.

Three conventional sources of dc current exist in the circuit and can contribute to $i_{m}$. First, an unintentional (drift) dc bias $V_{d}$ can produce a "drift" current $i_{d}$. Second, rectification of stray interference of frequency $\omega$ and magnitude $V_{\omega}$ can produce a rectified current $i_{r}$. Third, 


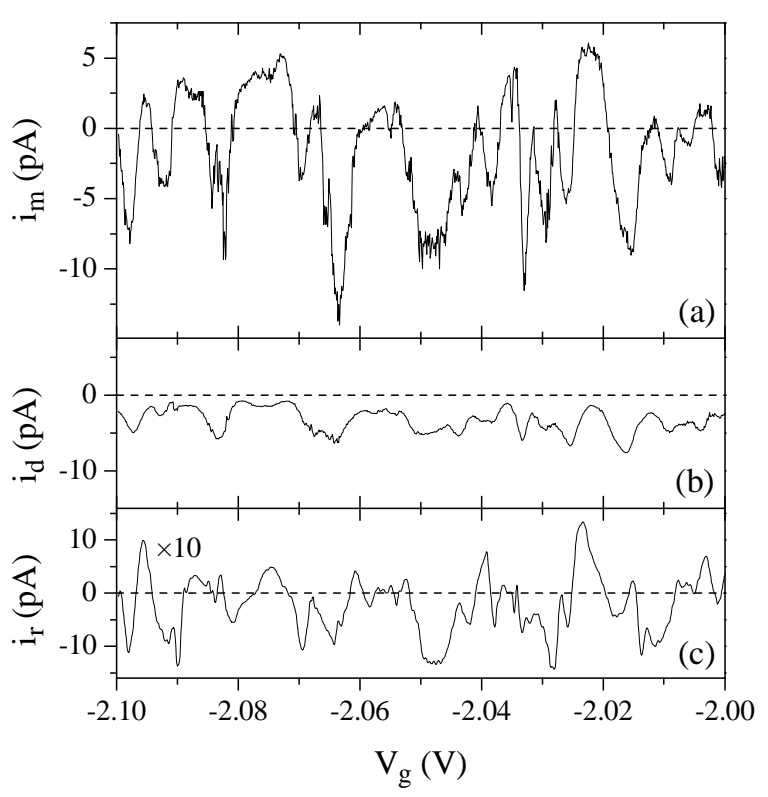

FIG. 2: (a) The measured dc current $i_{m}$ in the source-drain circuit with no applied bias within the range of $V_{g}$ highlighted in Fig. 1. (b,c) The contribution to $i_{m}$ from the drift current $i_{d}$ due to the presence of unintentional dc biases in the circuit (b), and from the rectified current $i_{r}$ due to the rectification of stray interference (c).

there is a small leakage current from the gate $i_{g}$, which splits into $(1-\alpha) i_{g}$ in the gate-source and $\alpha i_{g}$ in the gate-drain branches of the circuit. If we include an additional, unknown current $i_{0}$ as a fourth contribution, the total measured current $i_{m}$ can be written as:

$$
i_{m}=\frac{\mathrm{d} i}{\mathrm{~d} V} V_{d}+\frac{1}{4} \frac{\mathrm{d}^{2} i}{\mathrm{~d} V^{2}} \sum_{\omega \in \Omega} V_{\omega}^{2}+\alpha i_{g}+i_{0},
$$

where the first two terms on the right define $i_{d}$ and $i_{r}$ respectively. We have found that the conventional contributions do not constitute the primary element of the measured current $i_{m}$, either in the magnitude or fine structure, and instead $i_{0}$ dominates $i_{m}$. In order to investigate the nature of $i_{0}$, we first compare it to $i_{d}, i_{r}$ and $i_{g}$; then we propose a new mechanism of current generation that accounts for its existence.

The first contribution to $i_{m}$ is derived from an unintentional dc voltage in the source-drain circuit, which is due to the pre-amplifier. This voltage was found to change monotonically over the course of an experiment (1-2 hours) by $\sim 200 \mathrm{nV} /$ hour. In Fig. 2(b) the resultant drift current $i_{d}$ that can be ascribed to the average of this voltage, $V_{d}$, is shown (Eqn. 1). From this we see that the amplitude of $i_{d}$ is much smaller than the measured current, and more importantly, it is only driven in one direction. To quantify this difference, we calculate the correlation coefficient $C\left(i_{d}, i_{m}\right)=\left\langle\delta i_{d} \delta i_{m}\right\rangle /\left\langle\delta i_{d}^{2}\right\rangle^{1 / 2}\left\langle\delta i_{m}^{2}\right\rangle^{1 / 2}$, where $\delta i=i-\langle i\rangle$ and $\langle\cdots\rangle$ is an average over the gate voltage range shown in the figure. We find $C\left(i_{d}, i_{m}\right)=$ 0.61 , which increases to $>0.9$ when an intentional bias voltage $(\sim 1 \mathrm{mV})$ is applied such that $i_{d}$ dominates $i_{m}$. However, if all the conventional contributions are first subtracted from $i_{m}$ (see below) we find that $C\left(i_{d}, i_{0}\right)$ is only -0.28 . This low correlation, together with the small magnitude and singular direction shows conclusively that the mechanism associated with a dc bias voltage cannot account for the observed effect.

The second contribution to the measured current is rectification as a result of the non-linear nature of the system (as evinced in Fig. 1). The non-linearity of nanostructures has been observed previously, e.g. [7, 8, [9]. For such a system, the rectified current $i_{r}$ is related, through the second derivative $\mathrm{d}^{2} i / \mathrm{d} V^{2}\left(V_{g}\right)$, to the second harmonic response at frequency $2 \omega$ to an applied ac bias at $\omega$. In the absence of an applied ac bias, a rectified current is still present due to residual stray interference coupling to the circuit, predominantly the part outside the screen room. Therefore $i_{r}$ as a function of $V_{g}$ can be reconstructed from measurements of the second harmonic and the integral of the voltage noise across the channel. In Eqn. 1, the frequency range $\Omega$ for which $V_{\omega} \neq 0$ was found experimentally to have an upper limit of $20 \mathrm{kHz}$. The calculated rectified current is shown in Fig. 2(c), where it can be seen that it is approximately an order of magnitude smaller than $i_{m}$. If we also compare the correlation $C\left(i_{r}, i_{0}\right)=0.14$ with that obtained when a strong ac bias $\left(V_{\omega}>100 \mu \mathrm{V}\right)$ is intentionally applied to the channel, $C\left(i_{r}, i_{m}\right) \sim 1$, we conclude that $i_{0}$ cannot be related to the rectification of stray interference. We confirmed this in additional experiments where the measurement apparatus outside the screen room was replaced by analogue meters and batteries (to control $V_{g}$ ) mounted directly upon the refrigerator inside the room. The measurement of $i_{0}\left(V_{g}\right)$ by discrete points (the meters being read by candlelight) yielded the same result as that presented.

Figure 3 shows the spontaneous current $i_{0} \approx i_{m}-i_{d}-$ $i_{r}$. Also shown is the gate leakage current $i_{g}$. This is roughly constant $(\sim 2 \mathrm{pA})$ and its contribution to the measured current, determined by $\alpha \sim 0.5$, is small compared to $i_{0}$. In addition, it is important to note that $i_{0}$ flows around the source-drain circuit, whereas $i_{g}$ flows down each branch in the same direction, see Fig. 1(inset). The absence of fine structure in $i_{g}$ also shows that it cannot directly account for $i_{0}$, although in the model we propose it plays a key role in its generation.

It is interesting to note that samples defined by longer gate lengths, up to $2.0 \mu \mathrm{m}$, have shown no evidence in our experiments for producing spontaneous current. This suggests a critical dependence of its magnitude on the length of the channel. The model discussed below shows that indeed there can be an optimal channel length for the observation of the effect, which is close to that of our experiment. 


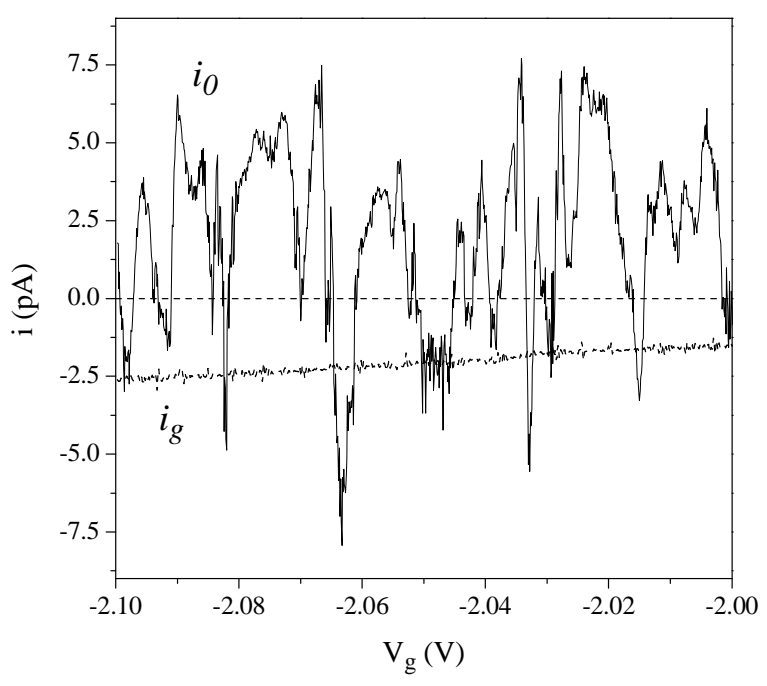

FIG. 3: The spontaneous current $i_{0}$, after subtracting the contributions of the drift and rectified currents from the measured current $i_{m}$ in Fig. 2. Also shown is the gate leakage current $i_{g}$ in the same range of gate voltage.

The model explains the experimental observations in terms of magnification of the gate leakage current. First we note that, although the leakage current is small, the power it supplies, $i_{g} V_{g} \sim 4 \cdot 10^{-12} \mathrm{~W}$, is enough to support the current $i_{0}$ in the source-drain circuit (in fact, it is significantly larger than the dissipated power $i_{0}^{2} R \sim 10^{-17} \mathrm{~W}$, where $R$ is the circuit resistance). Thus we do not have a situation of perpetuum mobile.

We suggest the following sequence of events (detailed

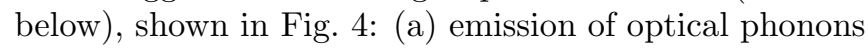
by electrons tunnelling from the gate; (b) conversion of optical into acoustic phonons; (c) excitation of "secondary" electrons by these acoustic phonons; (d) diffusion of secondary electrons into the contacts, and their subsequent return to the channel.

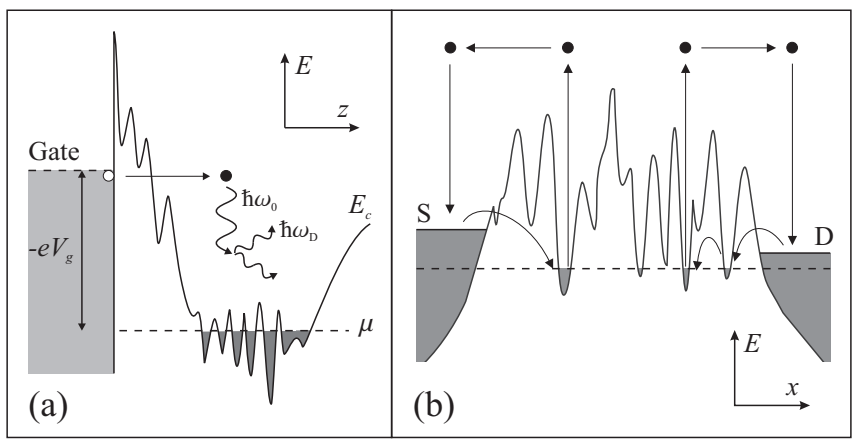

FIG. 4: The physical mechanism to explain the spontaneous current as a multistage process of relaxation and excitation. (a) The relaxation of hot electrons from the gate into the channel (Stages a-b, described in the text). (b) Diffusion and neutralization of secondary electrons from the channel (Stages c-d). The equilibrium Fermi level is shown as a dotted line. a. Electrons from the gate are injected into the channel with energy $\simeq e\left|V_{g}\right| \approx 2 \mathrm{eV}$ (Fig. 3), which is large compared with the sample temperature. So these electrons relax rapidly, predominantly by the emission of a cascade of $N_{p}=e V_{g} / \hbar \omega_{0} \gg 1$ optical phonons with energy $\hbar \omega_{0}$, either inside the channel or in the contacts close to the channel. The hot electrons mostly reside in the side valleys of GaAs with small mobility and large effective mass $0.35 \cdot 10^{-27} \mathrm{~g}[10]$, so their initial velocity can be roughly estimated as $v \sim 10^{8} \mathrm{~cm} / \mathrm{s}$ (from the condition $m v^{2} / 2 \approx 1 \mathrm{eV}$ ). Consequently, in a short channel (in our case $\sim 10^{-5} \mathrm{~cm}$ ) only a few optical phonons are emitted before the hot electron reaches a contact, where it continues to emit optical phonons. (The contacts are made of heavily doped GaAs with Fermi energy $\sim 10 \mathrm{meV}$, and in such material the emission of optical phonons remains the most efficient mechanism of electron energy relaxation [11.) The typical size of the contact region where the phonons are emitted is $L_{\mathrm{dif}}=\left(D N_{p} \tau_{\mathrm{e}-\mathrm{ph}}\right)^{1 / 2}$, where $D=v^{2} \tau_{p} / 3$ and $\tau_{p}$ (estimated below) is the elastic electron mean free time, while $\tau_{\mathrm{e}-\mathrm{ph}} \sim 10^{-13} \mathrm{~s}$ is the relaxation time due to emission of an optical phonon.

It is known that the room-temperature electron mobility in the side valley is $\sim 150 \mathrm{~cm}^{2} \mathrm{~V}^{-1} \mathrm{~s}^{-1}$, from which one estimates for an electron energy of $k_{B} T \sim 30 \mathrm{meV}$ that $\tau_{p} \approx 3 \cdot 10^{-14} \mathrm{~s}$. It is expected that scattering of hot electrons is mostly due to polar scattering by optical phonons 11] for which $\tau_{p} \propto \varepsilon^{1 / 2}$. Thus, for a typical energy $\sim 1 \mathrm{eV}$ the value of $\tau_{p}$ is about an order of magnitude larger than its equilibrium, room-temperature value. Taking into account an additional factor $\ln \left(\varepsilon / \hbar \omega_{0}\right)$ in the relaxation rate [11] we estimate $\tau_{p} \sim 10^{-13} \mathrm{~s}$. Consequently, an estimate for the penetration depth of a hot electron into the contact is $L_{\mathrm{dif}} \approx 1 \mu \mathrm{m}$.

$b$. Each optical phonon quickly decays into two highenergy acoustic phonons over the characteristic time $\tau_{\mathrm{op}} \sim 10^{-11} \mathrm{~s}[12]$. However, the decay of acoustic phonons is much weaker. The transverse modes practically do not decay, and their relaxation is mostly due to their conversion to longitudinal modes in the course of phonon-impurity scattering. One expects that the crosssection for the scattering of transverse acoustic phonons with $\hbar \omega_{D} \sim 15 \mathrm{meV}$ by impurities is of the order of the atomic one, $\sigma \sim 10^{-15} \mathrm{~cm}^{2}$. The mean free path for such phonons within the contacts at impurity concentration $N_{i} \sim 10^{17} \mathrm{~cm}^{-3}$ is $l=\left(\sigma N_{i}\right)^{-1} \sim 10^{-2} \mathrm{~cm}$, and so the majority of the phonons created over the distance of $L_{\mathrm{dif}}$ can easily (ballistically) reach the channel region. Thus, the number of acoustic phonons in the channel produced by one tunnelling electron is $\sim e V_{g} / 2 \hbar \omega_{D}$.

c. These phonons ionize donors in the channel creating "secondary" electrons. The probability for them to do so can be estimated using Fermi's golden rule, the 
squared matrix element being

$$
\frac{\lambda^{2} \hbar q}{M \omega_{q}}\left|\left\langle\frac{e^{-r / a}}{a^{3 / 2}}\left|\frac{e^{i \mathbf{q r}}}{\mathcal{V}^{1 / 2}}\right| \frac{e^{i \mathbf{k r}}}{\mathcal{V}^{1 / 2}}\right\rangle\right|^{2} \approx \frac{\lambda^{2} \hbar a}{M \omega_{q} q \mathcal{V}}
$$

Here $a$ is the localization length, $\mathbf{q}$ and $\mathbf{k}$ are the wave vectors of the phonon and excited electron, respectively; $\lambda$ is the deformation potential, $M$ the atomic mass, and $\mathcal{V}$ is the normalization volume. One calculates the phonon scattering rate due to the ionization processes as

$$
\frac{1}{\tau_{\mathrm{ph}, \mathrm{i}}} \sim \frac{\omega_{q} N_{i} a^{3}}{(q a)^{2}} \frac{\lambda^{2}}{E_{b}^{2}}\left(\frac{\hbar \omega_{q}-E_{i}}{E_{b}}\right)^{1 / 2},
$$

where $E_{i}$ is the donor ionization energy and $E_{b}$ is of the order of the atomic energy. Since $\lambda \sim E_{b}, q a \sim 10$, $\hbar \omega_{q}-E_{i} \sim 10^{-2} E_{b}$ and $N_{i} a^{3} \sim 1$, one has $\tau_{\mathrm{ph}, \mathrm{i}}^{-1} \sim 10^{-3} \omega_{q}$. Correspondingly, the mean free path with respect to ionization is about $3 \cdot 10^{-5} \mathrm{~cm}$.

From the above estimates it follows that the nonequilibrium acoustic phonons effectively relax within the channel via ionization of the donors. The net current of secondary electrons is thus $\sim\left(e V_{g} / 2 \hbar \omega_{D}\right) i_{g}$. The term in parentheses, which is $\sim 100$, can be regarded as an amplification factor for the gate leakage current. Experimentally it was shown above that the required magnification is $\lesssim 10$, which is well within the theoretical limit.

$d$. Since $\hbar \omega_{D} \sim 15 \mathrm{meV}>E_{i}$, the secondary electrons have a large characteristic energy and a correspondingly large velocity, $\sim(2-4) \cdot 10^{7} \mathrm{~cm} / \mathrm{s}$, to escape from the initial donor. The energy of these electrons is well above the conduction band edge, thus they are only weakly sensitive to the potential landscape of the channel. This fact ensures that only a small difference exists in the flow of secondary electrons towards the two contacts. For a characteristic electron energy around $10 \mathrm{meV}$ and mean free time $10^{-12} \mathrm{~s}$ (estimated for the Coulomb scattering by charged impurities with concentration $N_{i}$ ) the mean free path is $\sim(2-4) \cdot 10^{-5} \mathrm{~cm}$, which is of the order of the length of the channel. Hence most of the secondary electrons reach the contacts ballistically where they relax by electron-electron interaction.

The system now needs to restore quasi-neutrality, and the only way to do so is for electrons to hop back to the channel and be captured by the ionized donors. Although the secondary electrons diffuse equally to both contacts, their return to the donors is asymmetric. This is due to the fact that the channel is mesoscopic, and the hopping paths from the two contacts are different, Fig. 4(b). As a result, the electrochemical potentials in the contacts are increased differently with respect to the equilibrium Fermi level. It is this potential difference that drives the current $i_{0}$ in the external circuit. In experiment the degree of asymmetry is controlled by the gate voltage which determines the spatial position of donors in the channel. Thus the magnitude and direction of $i_{0}$ is critically dependent upon its value, Fig. 3.

Our estimations above show that the proposed mechanism is indeed realized in systems with channel length $0.1 \mu \mathrm{m}$; moreover this length appears to be an optimal one for its realization. Upon increasing the length the effect is significantly suppressed, both due to the increased probability for secondary electrons to relax directly back to the ionized donors in the channel (Stage d), and due to the decrease in the asymmetry of the channel. Contrarily, in shorter channels the process of ionization (Stage c) will be less efficient.

In conclusion we have observed a novel "phononelectric" effect in a gated nanostructure, which is seen as a spontaneous generation of a dc current with no driving voltage applied. Our explanation is based on the combination of leakage current magnification mediated by phonons and asymmetry in the channel controlled by the gate voltage.

AKS is grateful to V. Pokrovskii, V. I. Falko, M. Entin, M. E. Raikh, and A. O. Orlov for useful discussions. This work was supported by the EPSRC (U.K.) and the U.S. Department of Energy Office of Science through contract No. W-31-109-ENG-38.

[1] P. A. Lee and A. D. Stone, Phys. Rev. Lett. 55, 1622 (1985).

[2] B. L. Altshuler, JETP Lett 41, 648 (1985).

[3] V. I. Fal'ko and D. E. Khmel'nitskii, Sov. Phys. JETP 68, 186 (1989).

[4] A. A. Bykov, G. M. Gusev, Z. D. Kvon, D. I. Lubyshev, and V. P. Migal, JETP Lett. 49, 13 (1989).

[5] V. G. Veselago, V. N. Zavaritskii, M. S. Nunuparov, and A. B. Berkut, JETP Lett. 44, 490 (1986).

[6] P. S. Kop'ev, M. Y. Nadtochii, and V. M. Ustinov, Sov. Phys. Semicond. 23, 694 (1989).

[7] E. I. Laiko, A. O. Orlov, A. K. Savchenko, E. A. Ilichev, and E. A. Poltoratskii, Sov. Phys. JETP 66, 1258 (1987).

[8] R. Taboryski, A. K. Geim, M. Persson, and P. E. Lindelof, Phys. Rev. B 49, 7813 (1994).

[9] H. Linke, T. E. Humphrey, A. Lofgren, A. O. Sushkov, R. Newbury, R. P. Taylor, and P. Omling, Science 286, 2314 (1999).

[10] R. A. Smith, Semiconductors (Cambridge University Press, Cambridge, England, 1978), 2nd ed.

[11] V. F. Gantmakher and Y. B. Levinson, Carrier Scattering in Metals and Semiconductors (North-Holland, 1987).

[12] B. Danilchenko, A. Klimashov, S. Roshko, and M. Asche, Phys. Rev. B 50, 5725 (1994). 